\title{
Strong convergence theorems on a viscosity approximation method for a finite family of pseudo-contractive mappings in Banach spaces
}

\section{Yan Tang*}

${ }^{*}$ Correspondence:

ttyy7999@163.com

College of Mathematics and

Statistics, Chongqing Technology

and Business University, Chongqing, 400067, China

\begin{abstract}
In this paper, a new viscosity iterative process, which converges strongly to a common element of the set of fixed points of a finite family of pseudo-contractive mappings more general than non-expansive mappings, is introduced in Banach spaces. Strong convergence theorems are obtained under milder conditions. The results presented in this paper extend and unify most of the results that have been proposed for this class of nonlinear mappings.

MSC: 47H09; 47H10; 47L25
\end{abstract}

Keywords: pseudo-contractive mappings; fixed point; viscosity approximation; strong convergence

\section{Introduction}

Let $E$ be a real Banach space with dual $E^{*}$. A normalized duality mapping $J: E \rightarrow 2^{E^{*}}$ is defined by

$$
J x=\left\{f^{*} \in E^{*}:\left\langle x, f^{*}\right\rangle=\|x\|^{2}=\left\|f^{*}\right\|^{2}\right\}
$$

where $\langle\cdot, \cdot\rangle$ denotes the generalized duality pairing. It is well known that $E$ is smooth if and only if $J$ is single-valued, and if $E$ is uniformly smooth, then $J$ is uniformly continuous on bounded subsets of $E$.

Let $C$ be a closed convex subset of the Banach space $E$. A mapping $T: C \rightarrow C$ is called non-expansive if

$$
\|T x-T y\| \leq\|x-y\|, \quad \forall x, y \in C .
$$

A mapping $T$ is said to be pseudo-contractive if for any $x, y \in D(T)$, there exists $j(x-y) \in$ $J(x-y)$ such that

$$
\langle T x-T y, j(x-y)\rangle \leq\|x-y\|^{2} .
$$

O2014 Tang; licensee Springer. This is an Open Access article distributed under the terms of the Creative Commons Attribution License (http://creativecommons.org/licenses/by/2.0), which permits unrestricted use, distribution, and reproduction in any medium, provided the original work is properly cited. 
A mapping $T$ is said to be $\kappa$-strictly pseudo-contractive if for any $x, y \in D(T)$, there exist $j(x-y) \in J(x-y)$ and a constant $0 \leq \kappa \leq 1$ such that

$$
\langle x-y-(T x-T y), j(x-y)\rangle \geq \kappa\|(I-T) x-(I-T) y\|^{2} .
$$

Clearly, the class of pseudo-contractive mappings includes the class of strict pseudocontractive mappings and non-expansive mappings. We denote by $F(T)$ the set of fixed points of $T$, that is, $F(T)=\{x \in C: T x=x\}$.

An operator $A: C \rightarrow E$ is called accretive if there exists $j(x-y) \in J(x-y)$ such that

$$
\langle A x-A y, j(x-y)\rangle \geq 0, \quad \forall x, y \in C .
$$

We observe that $A$ is accretive if and only if $T:=I-A$ is pseudo-contractive, where $I$ is the identity mapping on $C$, and thus a zero of $A, N(A):=\{x \in D(A): A x=0\}$, is a fixed point of $T$. It is now well known that if $A$ is accretive, then the solutions of the equation $A x=0$ correspond to the equilibrium points of some evolution systems. Consequently, considerable research efforts have been devoted to iterative methods for approximating fixed points of $T$ when $T$ is pseudo-contractive (see, e.g., [1-3] and the references contained therein). A mapping $f: C \rightarrow C$ is called contractive with a contraction coefficient if there exists a constant $\rho \in(0,1)$ such that

$$
\|f(x)-f(y)\| \leq \rho\|x-y\|, \quad \forall x, y \in C .
$$

For finding an element of the set of fixed points of non-expansive mappings, Halpern [4] was the first to study the convergence of the scheme in 1967:

$$
x_{n+1}=\alpha_{n+1} u+\left(1-\alpha_{n+1}\right) T\left(x_{n}\right) .
$$

Viscosity approximation methods are very important because they are applied to convex optimization, linear programming, monotone inclusions and elliptic differential equations. In a Hilbert space, many authors have studied fixed point problems for pseudocontractive mappings by the viscosity approximation methods and obtained a series of good results (see [1-3, 5-18]).

In 2000, Moudafi [19] introduced viscosity approximation methods and proved the strong convergence of the following iterative algorithm in a Hilbert space under some suitable conditions:

$$
x_{n+1}=\alpha_{n} f\left(x_{n}\right)+\left(1-\alpha_{n}\right) T\left(x_{n}\right) .
$$

Moudafi [19] generalized Halpern's theorems in the direction of viscosity approximations.

In 2008, Yao et al. [6] proposed the following modified Mann iterations for nonexpansive mappings:

$$
\left\{\begin{array}{l}
y_{n}=\beta_{n} x_{n}+\left(1-\beta_{n}\right) T x_{n}, \\
x_{n+1}=\alpha_{n} f\left(x_{n}\right)+\left(1-\alpha_{n}\right) y_{n}, \quad \forall n \geq 0,
\end{array}\right.
$$

and obtained strong convergence theorems for a common fixed point of non-expansive mappings. 
Recently, Zegeye [20] introduced the following algorithm:

$$
x_{n+1}=\alpha_{n} f\left(x_{n}\right)+\left(1-\alpha_{n}\right) T_{r_{n}} F_{r_{n}} x_{n},
$$

where $T_{r_{n}}, F_{r_{n}}$ are non-expansive mappings, and obtained a strong convergence theorem but still in a Hilbert space.

On the other hand, for obtaining strong convergence theorems for a family of finite nonexpansive mappings, Takahashi [12] defined the following mapping $W_{n}$ :

$$
\left\{\begin{array}{l}
U_{n, 1}=\alpha_{n, 1} T_{1}+\left(1-\alpha_{n, 1}\right) I, \\
U_{n, 2}=\alpha_{n, 2} T_{2} U_{n, 1}+\left(1-\alpha_{n, 2}\right) I, \\
\cdots \\
W_{n}:=U_{n, m}=\alpha_{n, m} T_{m} U_{n, m-1}+\left(1-\alpha_{n, m}\right) I,
\end{array}\right.
$$

where $\left\{T_{i}, i=1,2, \ldots, m\right\}$ are non-expansive mappings, and $F\left(W_{n}\right)=\bigcap_{i=1}^{m} F\left(T_{i}\right)$.

Our concern now is the following: Is it possible to construct a new sequence in Banach spaces which converges strongly to a common element of fixed points of a finite family of pseudo-contractive mappings?

In this paper, motivated and inspired by the above results, we introduce a new iteration scheme in Banach spaces which converges strongly to a common element of the set of fixed points of continuous pseudo-contractive mappings more general than nonexpansive mappings. This provides affirmative answer to the above concern. Our theorems extend and unify most of the results that have been proposed for this class of nonlinear mappings.

\section{Preliminaries}

Let $E$ be a real Banach space with dual $E^{*}, C$ be a closed convex subset of $E$. Let $S=\{x \in E$ : $\|x\|=1\}$ denote the unit sphere of $E$. The space $E$ is said to have a Gâteaux differentiable norm if the $\operatorname{limit}_{t \rightarrow 0} \frac{\|x+t y\|-\|x\|}{t}$ exists for each $x, y \in S$ and in this case $E$ is said to be smooth. $E$ is said to be uniformly Gâteaux differentiable if for each $y \in S$, the limit above is uniformly attained for $x \in S$.

In the proof of our main results, we also need the following definitions and results.

Let $\mu$ be a continuous linear functional on $l^{\infty}$ satisfying $\|\mu\|=1=\mu(1)$. Then we know that $\mu$ is a mean on $N$ if and only if

$$
\inf \left\{a_{n} ; n \in N\right\} \leq \mu(a) \leq \sup \left\{a_{n} ; n \in N\right\}, \quad \forall a=\left(a_{1}, a_{2}, \ldots\right) \in l^{\infty} .
$$

According to time and circumstances, we use $\mu_{n}\left(a_{n}\right)$ instead of $\mu(a)$. A mean $\mu$ on $N$ is called a Banach limit if $\mu_{n}\left(a_{n}\right)=\mu_{n}\left(a_{n+1}\right)$ for every $a=\left(a_{1}, a_{2}, \ldots\right) \in l^{\infty}$.

Define a map $\varphi: E \rightarrow \mathbb{R}$ by $\varphi(y)=\mu_{n}\left\|x_{n}-y\right\|^{2},\left\{x_{n}\right\} \subset E$ is an arbitrary bounded sequence, then $\varphi(y)$ is convex and continuous, and $\varphi(y) \rightarrow \infty$ as $\|y\| \rightarrow \infty$. If $E$ is reflexive, there exists $z \in C$ such that $\varphi(z)=\inf _{y \in C} \varphi(y)$ (see [21]). So the set

$$
C_{\min }=\left\{z \in C ; \varphi(z)=\inf _{y \in C} \varphi(y)\right\} \neq \varnothing
$$

Clearly, $C_{\min }$ is a closed convex subset of $E$. 
In the sequel, we shall use the following lemmas.

Lemma 2.1 [7,22] Let $C$ be a nonempty closed convex subset of a Banach space $E$ with a uniformly Gâteaux differentiable norm. Let $\left\{x_{n}\right\}$ be a bounded sequence of $E$, and let $\mu_{n}$ be a Banach limit and $z \in C$. Then

$$
\mu_{n}\left\|x_{n}-z\right\|^{2}=\min _{y \in C} \mu_{n}\left\|x_{n}-y\right\|^{2}
$$

if and only if

$$
\mu_{n}\left\langle y-z, J\left(x_{n}-z\right)\right\rangle \leq 0, \quad \forall y \in C .
$$

Lemma 2.2 $[6,7]$ Let $\alpha$ be a real number and $\left(x_{0}, x_{1}, \ldots\right) \in l^{\infty}$ for all Banach limits satisfying $\mu_{n} x_{n} \leq \alpha$. If $\lim \sup _{n \rightarrow \infty}\left(x_{n+1}-x_{n}\right) \leq 0$, then $\limsup _{n \rightarrow \infty} x_{n} \leq \alpha$.

Lemma 2.3 [8] Let $\left\{a_{n}\right\}$ be a sequence of nonnegative real numbers satisfying the following relation:

$$
a_{n+1} \leq\left(1-\theta_{n}\right) a_{n}+\sigma_{n}, \quad n \geq 0,
$$

where $\left\{\theta_{n}\right\}$ is a sequence in $(0,1)$ and $\left\{\sigma_{n}\right\}$ is a real sequence such that

(i) $\sum_{n=0}^{\infty} \theta_{n}=\infty$;

(ii) $\lim \sup _{n \rightarrow \infty} \frac{\sigma_{n}}{\theta_{n}} \leq 0$ or $\sum_{n=0}^{\infty} \sigma_{n}<\infty$.

Then $\lim _{n \rightarrow \infty} a_{n}=0$.

Lemma 2.4 [10] Let $\left\{x_{n}\right\}$ and $\left\{z_{n}\right\}$ be bounded sequences in a Banach space, and let $\left\{\beta_{n}\right\}$ be a sequence in $[0,1]$ which satisfies the following condition:

$$
0<\liminf _{n \rightarrow \infty} \beta_{n}<\limsup _{n \rightarrow \infty} \beta_{n}<1 .
$$

Suppose that

$$
x_{n+1}=\beta_{n} x_{n}+\left(1-\beta_{n}\right) z_{n}, \quad n \geq 0,
$$

and

$$
\lim _{n \rightarrow \infty}\left(\left\|z_{n+1}-z_{n}\right\|-\left\|x_{n+1}-x_{n}\right\|\right) \leq 0 .
$$

Then $\lim _{n \rightarrow \infty}\left\|z_{n}-x_{n}\right\|=0$.

Lemma 2.5 [23] Let $E$ be a real Banach space with dual $E^{*}, J: E \rightarrow 2^{E^{*}}$ be the generalized duality pairing, then, $\forall x, y \in E$,

$$
\|x+y\|^{2} \leq\|x\|^{2}+2\langle y, j(x+y)\rangle, \quad \forall j(x+y) \in J(x+y) .
$$

Moreover, by a similar argument as in the proof of Lemmas 3.1 and 3.2 of [24], we get the following lemmas. 
Lemma 2.6 Let $C$ be a nonempty closed convex subset of a uniformly smooth strictly convex real Banach space E. Let $T: C \rightarrow E$ be a continuous pseudo-contractive mapping. Then, for $r>0$ and $x \in E$, there exists $z \in C$ such that

$$
\langle j(y-z), T z\rangle-\frac{1}{r}\langle j(y-z),(1+r) z-x\rangle \leq 0, \quad \forall y \in C .
$$

Proof Let $x \in C$ and $r>0$. Let $A:=I-T$, clearly $A$ is a continuous accretive mapping. Thus, by a similar argument as in [24], the lemma holds.

Lemma 2.7 Let $C$ be a nonempty closed convex subset of a uniformly smooth strictly convex real Banach space E. Let $T: C \rightarrow E$ be a continuous pseudo-contractive mapping, define the mapping $T_{r}$ as follows: $x \in E, r \in(0, \infty)$

$$
T_{r}(x)=\left\{z \in C:\langle j(y-z), T z\rangle-\frac{1}{r}\langle j(y-z),(1+r) z-x\rangle \leq 0, \forall y \in C\right\} .
$$

Then the following hold:

(i) $T_{r}$ is single-valued;

(ii) $T_{r}$ is a non-expansive mapping;

(iii) $F\left(T_{r}\right)=F(T)$;

(iv) $F(T)$ is closed and convex.

Proof Let $A:=I-T$, we note that $A$ is a continuous accretive mapping and that $\langle j(y-z)$, $T z\rangle-\frac{1}{r}\langle j(y-z),(1+r) z-x\rangle \leq 0$ is equivalent to $\langle j(y-z), A z\rangle+\frac{1}{r}\langle j(y-z), z-x\rangle \geq 0$. Thus, by a similar argument as in [24], the conclusions of (i)-(iv) hold.

\section{Main results}

Let $C$ be a nonempty, closed and convex subset of a smoothly, strictly convex and reflexive real Banach space $E$ with dual $E^{*}$. Let $\left\{T_{i}: C \rightarrow C, i=1,2, \ldots, m\right\}$ be a finite family of continuous pseudo-contractive mappings. For the rest of this article, $T_{i r_{n}} x$ and $W_{n}$ are defined as follows: for $x \in E, r_{n} \in(0, \infty)$,

$$
\begin{aligned}
& T_{i r_{n}}(x):=\left\{z \in C:\left\langle j(y-z), T_{i} z\right\rangle-\frac{1}{r_{n}}\left\langle j(y-z),\left(1+r_{n}\right) z-x\right\rangle \leq 0, \forall y \in C\right\}, \\
& \left\{\begin{array}{l}
U_{n, 1}=\alpha_{n, 1} T_{1 r_{n}}+\left(1-\alpha_{n, 1}\right) I, \\
U_{n, 2}=\alpha_{n, 2} T_{2 r_{n}} U_{n, 1}+\left(1-\alpha_{n, 2}\right) I, \\
\ldots \\
W_{n}:=U_{n, m}=\alpha_{n, m} T_{m r_{n}} U_{n, m-1}+\left(1-\alpha_{n, m}\right) I .
\end{array}\right.
\end{aligned}
$$

We know from Lemma 2.7 and Takahashi [12] that $T_{i r_{n}}$ and $W_{n}$ are firmly non-expansive mappings and $F\left(W_{n}\right)=\bigcap_{i=1}^{m} F\left(T_{i r_{n}}\right)=\bigcap_{i=1}^{m} F\left(T_{i}\right)$. Denote $F:=F\left(W_{n}\right)$.

Theorem 3.1 Let $C$ be a nonempty closed convex subset of a uniformly smooth strictly convex real Banach space E. Let $\left\{T_{i}: C \rightarrow C, i=1,2, \ldots, m\right\}$ be a finite continuous pseudocontractive mapping, for each bounded sequence $x_{n}$ and for each Banach limit $\mu_{n}, C_{\min }$ is defined as (2.2) satisfying $F \cap C_{\min } \neq \varnothing$. Let $f: C \rightarrow C$ be a contraction with a contraction 
coefficient $\rho \in(0,1)$. The mappings $T_{i r_{n}}$ and $W_{n}$ are defined as (3.1) and (3.2), respectively. Let $\left\{x_{n}\right\}$ be a sequence generated by $x_{0} \in C$ :

$$
\left\{\begin{array}{l}
y_{n}=\lambda_{n} x_{n}+\left(1-\lambda_{n}\right) W_{n} x_{n}, \\
x_{n+1}=\alpha_{n} f\left(x_{n}\right)+\beta_{n} x_{n}+\gamma_{n} y_{n},
\end{array}\right.
$$

where $\lambda_{n} \in[0,1],\left\{\alpha_{n}\right\},\left\{\beta_{n}\right\},\left\{\gamma_{n}\right\}$ are sequences of nonnegative real numbers in $[0,1]$ and

(i) $\alpha_{n}+\beta_{n}+\gamma_{n}=1, n \geq 0$;

(ii) $\lim _{n \rightarrow \infty} \alpha_{n}=0, \sum_{n=1}^{\infty} \alpha_{n}=\infty$;

(iii) $0<\liminf _{n \rightarrow \infty} \lambda_{n} \leq \lim \sup _{n \rightarrow \infty} \lambda_{n}<1$;

(iv) $\lim _{n \rightarrow \infty}\left(\alpha_{n, i}-\alpha_{n-1, i}\right)=0 ; \lim _{n \rightarrow \infty}\left|r_{n+1}-r_{n}\right|=0$.

Then the sequence $\left\{x_{n}\right\}$ converges strongly to a common fixed point of $\left\{T_{i}: C \rightarrow C, i=\right.$ $1,2, \ldots, m\}$.

Proof First we prove that $\left\{x_{n}\right\}$ is bounded. Take $p \in F \cap C_{\min }$, because $W_{n}$ is non-expansive, then we have that

$$
\left\|y_{n}-p\right\| \leq \lambda_{n}\left\|x_{n}-p\right\|+\left(1-\lambda_{n}\right)\left\|W_{n} x_{n}-W_{n} p\right\| \leq\left\|x_{n}-p\right\| .
$$

For $n \geq 0$, because $f$ is contractive, we have from (3.4) that

$$
\begin{aligned}
\left\|x_{n+1}-p\right\| & =\left\|\alpha_{n}\left(f\left(x_{n}\right)-p\right)+\beta_{n}\left(x_{n}-p\right)+\gamma_{n}\left(y_{n}-p\right)\right\| \\
& \leq \alpha_{n}\left\|f\left(x_{n}\right)-f(p)\right\|+\alpha_{n}\|f(p)-p\|+\beta_{n}\left\|x_{n}-p\right\|+\gamma_{n}\left\|y_{n}-p\right\| \\
& \leq \rho \alpha_{n}\left\|x_{n}-p\right\|+\alpha_{n}\|f(p)-p\|+\left(1-\alpha_{n}\right)\left\|x_{n}-p\right\| \\
& \leq\left[1-(1-\rho) \alpha_{n}\right]\left\|x_{n}-p\right\|+\alpha_{n}\|f(p)-p\| \\
& \leq \max \left\{\left\|x_{0}-p\right\|, \frac{f(p)-p}{1-\rho}\right\} .
\end{aligned}
$$

Therefore, $\left\{x_{n}\right\}$ is bounded. Consequently, we get that $\left\{W_{n} x_{n}\right\}$ and $\left\{y_{n}\right\},\left\{f\left(x_{n}\right)\right\}$ are bounded.

Next, we show that $\left\|x_{n+1}-x_{n}\right\| \rightarrow 0$. Let $x_{n+1}=\beta_{n} x_{n}+\left(1-\beta_{n}\right) z_{n}$. Hence we have that

$$
\begin{aligned}
z_{n+1}-z_{n}= & \frac{\alpha_{n+1}}{1-\beta_{n+1}}\left(f\left(x_{n+1}\right)-f\left(x_{n}\right)\right)+\left(\frac{\alpha_{n+1}}{1-\beta_{n+1}}-\frac{\alpha_{n}}{1-\beta_{n}}\right) f\left(x_{n}\right) \\
& +\frac{\gamma_{n+1}}{1-\beta_{n+1}}\left(y_{n+1}-y_{n}\right)+\left(\frac{\gamma_{n+1}}{1-\beta_{n+1}}-\frac{\gamma_{n}}{1-\beta_{n}}\right) y_{n} .
\end{aligned}
$$

Because $y_{n}=\lambda_{n} x_{n}+\left(1-\lambda_{n}\right) W_{n} x_{n}$, so we have that

$$
\begin{aligned}
& \left\|y_{n+1}-y_{n}\right\| \\
& \quad \leq \lambda_{n+1}\left\|x_{n+1}-x_{n}\right\|+\left(1-\lambda_{n+1}\right)\left\|W_{n+1} x_{n+1}-W_{n} x_{n}\right\|+\left|\lambda_{n+1}-\lambda_{n}\right|\left\|x_{n}-W_{n} x_{n}\right\| \\
& \quad \leq\left\|x_{n+1}-x_{n}\right\|+\left(1-\lambda_{n+1}\right)\left\|W_{n+1} x_{n}-W_{n} x_{n}\right\|+\left|\lambda_{n+1}-\lambda_{n}\right|\left\|x_{n}-W_{n} x_{n}\right\| \\
& \quad \leq\left\|x_{n+1}-x_{n}\right\|+\left\|W_{n+1} x_{n}-W_{n} x_{n}\right\|+\left|\lambda_{n+1}-\lambda_{n}\right|\left\|x_{n}-W_{n} x_{n}\right\| .
\end{aligned}
$$


Because $T_{i r_{n}}$ and $U_{n, m}$ are non-expansive mappings, we have from (3.2) that

$$
\begin{aligned}
\left\|W_{n+1} x_{n}-W_{n} x_{n}\right\| \\
=\left\|\alpha_{n+1, m} T_{m r_{n+1}} U_{n+1, m-1} x_{n}+\left(\alpha_{n, m}-\alpha_{n+1, m}\right) x_{n}-\alpha_{n, m} T_{m r_{n}} U_{n, m-1} x_{n}\right\| \\
\leq\left|\alpha_{n+1, m}-\alpha_{n, m}\right|\left\{\left\|x_{n}\right\|+\left\|T_{m r_{n}} U_{n, m-1} x_{n}\right\|\right\} \\
\quad+\alpha_{n+1, m}\left\|T_{m r_{n+1}} U_{n+1, m-1} x_{n}-T_{m r_{n}} U_{n, m-1} x_{n}\right\| \\
\leq 2 M\left|\alpha_{n+1, m}-\alpha_{n, m}\right|+\alpha_{n+1, m}\left\|T_{m r_{n+1}} U_{n+1, m-1} x_{n}-T_{m r_{n}} U_{n, m-1} x_{n}\right\| \\
\leq 2 M\left|\alpha_{n+1, m}-\alpha_{n, m}\right|+\left\|T_{m r_{n+1}} U_{n+1, m-1} x_{n}-T_{m r_{n}} U_{n, m-1} x_{n}\right\|
\end{aligned}
$$

where $M=\max \left\{\left\|x_{n}\right\|, \sup _{0 \leq i \leq m-2}\left\|T_{m-i, r_{n}} U_{n, m-(i+1)} x_{n}\right\|\right\}$.

Let $u_{n}=T_{m r_{n}} v_{n}, u_{n+1}=T_{m r_{n+1}} v_{n+1}, v_{n}=U_{n, m-1} x_{n}, v_{n+1}=U_{n+1, m-1} x_{n}$, by the definition of mapping $T_{i r_{n}}$, we have that

$$
\begin{aligned}
& \left\langle j\left(y-u_{n}\right), T_{m} u_{n}\right\rangle-\frac{1}{r_{n}}\left\langle j\left(y-u_{n}\right),\left(1+r_{n}\right) u_{n}-v_{n}\right\rangle \leq 0, \quad \forall y \in C, \\
& \left\langle j\left(y-u_{n+1}\right), T_{m} u_{n+1}\right\rangle-\frac{1}{r_{n+1}}\left\langle j\left(y-u_{n+1}\right),\left(1+r_{n+1}\right) u_{n+1}-v_{n+1}\right\rangle \leq 0, \quad \forall y \in C .
\end{aligned}
$$

Let $y:=u_{n+1}$ in (3.8), and let $y:=u_{n}$ in (3.9), we have that

$$
\begin{aligned}
& \left\langle j\left(u_{n+1}-u_{n}\right), T_{m} u_{n}\right\rangle-\frac{1}{r_{n}}\left\langle j\left(u_{n+1}-u_{n}\right),\left(1+r_{n}\right) u_{n}-v_{n}\right\rangle \leq 0, \\
& \left\langle j\left(u_{n}-u_{n+1}\right), T_{m} u_{n+1}\right\rangle-\frac{1}{r_{n+1}}\left\langle j\left(u_{n}-u_{n+1}\right),\left(1+r_{n+1}\right) u_{n+1}-v_{n+1}\right\rangle \leq 0 .
\end{aligned}
$$

Adding (3.10) and (3.11), and because $T_{m}$ is pseudo-contractive, we have that

$$
\left\langle j\left(u_{n+1}-u_{n}\right), \frac{u_{n}-v_{n}}{r_{n}}-\frac{u_{n+1}-v_{n+1}}{r_{n+1}}\right\rangle \geq 0 .
$$

Therefore we have

$$
\left\langle j\left(u_{n+1}-u_{n}\right), u_{n}-v_{n}-\frac{r_{n}\left(u_{n+1}-v_{n+1}\right)}{r_{n+1}}+u_{n+1}-u_{n+1}\right\rangle \geq 0 .
$$

Without loss of generality, let $b$ be a real number such that $r_{n}>b>0, \forall n \in N$, hence we have that

$$
\left\|u_{n+1}-u_{n}\right\| \leq\left\|v_{n+1}-v_{n}\right\|+\frac{1}{b}\left|r_{n+1}-r_{n}\right| M_{1}
$$

where $M_{1}=\sup _{2 \leq i \leq m}\left\{\left\|T_{i, r_{n}} U_{n, i-1} x_{n}\right\|+\left\|U_{n, i-1} x_{n}\right\|\right\}$.

Since $v_{n}=U_{n, m-1} x_{n}, v_{n+1}=U_{n+1, m-1} x_{n}$, so we have that

$$
\begin{aligned}
& \left\|v_{n+1}-v_{n}\right\| \\
& \quad=\left\|\alpha_{n+1, m-1} T_{m-1 r_{n+1}} U_{n+1, m-2} x_{n}+\left(\alpha_{n, m-1}-\alpha_{n+1, m-1}\right) x_{n}-\alpha_{n, m-1} T_{m-1 r_{n}} U_{n, m-2} x_{n}\right\| \\
& \quad \leq\left|\alpha_{n+1, m-1}-\alpha_{n, m-1}\right|\left\{\left\|x_{n}\right\|+\left\|T_{m-1 r_{n}} U_{n, m-2} x_{n}\right\|\right\}
\end{aligned}
$$




$$
\begin{aligned}
& +\alpha_{n+1, m-1}\left\|T_{m-1 r_{n+1}} U_{n+1, m-2} x_{n}-T_{m-1 r_{n}} U_{n, m-2} x_{n}\right\| \\
\leq & 2 M\left|\alpha_{n+1, m-1}-\alpha_{n, m-1}\right|+\alpha_{n+1, m-1}\left\|T_{m-1 r_{n+1}} U_{n+1, m-2} x_{n}-T_{m-1 r_{n}} U_{n, m-2} x_{n}\right\| .
\end{aligned}
$$

By the definition of $T_{i r_{n}}$, repeating steps from (3.8) to (3.12), we have that

$$
\begin{aligned}
& \left\|T_{m-1 r_{n+1}} U_{n+1, m-2} x_{n}-T_{m-1 r_{n}} U_{n, m-2} x_{n}\right\| \\
& \quad \leq\left\|U_{n+1, m-2} x_{n}-U_{n, m-2} x_{n}\right\|+\frac{1}{b}\left|r_{n+1}-r_{n}\right| M_{1} .
\end{aligned}
$$

Consequently, we have from (3.12) and (3.13), (3.14) that

$$
\begin{aligned}
\| u_{n+1}- & u_{n} \| \\
\leq & 2 M\left|\alpha_{n+1, m-1}-\alpha_{n, m-1}\right|+\alpha_{n+1, m-1}\left\{\left\|U_{n+1, m-2} x_{n}-U_{n, m-2} x_{n}\right\|+\frac{1}{b}\left|r_{n+1}-r_{n}\right| M_{1}\right\} \\
& \quad+\frac{1}{b}\left|r_{n+1}-r_{n}\right| M_{1} \\
\leq & 2 M\left|\alpha_{n+1, m-1}-\alpha_{n, m-1}\right|+\left\|U_{n+1, m-2} x_{n}-U_{n, m-2} x_{n}\right\|+\frac{2}{b}\left|r_{n+1}-r_{n}\right| M_{1} \\
\leq & \ldots \\
\leq & 2 M \sum_{i=2}^{m-1}\left|\alpha_{n+1, i}-\alpha_{n, i}\right|+\frac{m-1}{b}\left|r_{n+1}-r_{n}\right| M_{1}+\left\|U_{n+1,1} x_{n}-U_{n, 1} x_{n}\right\| .
\end{aligned}
$$

From (3.2) we have that

$$
\begin{aligned}
\left\|U_{n+1,1} x_{n}-U_{n, 1} x_{n}\right\| & =\left\|\left(\alpha_{n, 1}-\alpha_{n+1,1}\right) x_{n}+\alpha_{n+1,1} T_{1 r_{n+1}} x_{n}-\alpha_{n, 1} T_{1 r_{n}} x_{n}\right\| \\
& \leq 2 M\left|\alpha_{n+1,1}-\alpha_{n, 1}\right|+\left\|T_{1 r_{n+1}} x_{n}-T_{1 r_{n}} x_{n}\right\| .
\end{aligned}
$$

By the definition of $T_{i r_{n}}$, repeating steps from (3.8) to (3.12), we have that

$$
\left\|T_{1 r_{n+1}} x_{n}-T_{1 r_{n}} x_{n}\right\| \leq \frac{\left|r_{n+1}-r_{n}\right|}{b} M_{2}
$$

where $M_{2}=\max \left\{M_{1}, \sup \left\{\left\|T_{1 r_{n}} x_{n}\right\|+\left\|x_{n}\right\|\right\}\right\}$. Substituting (3.17) into (3.16), (3.16) into (3.15), (3.15) into (3.7), we have that

$$
\left\|W_{n+1} x_{n}-W_{n} x_{n}\right\| \leq 2 M \sum_{i=1}^{m}\left|\alpha_{n+1, i}-\alpha_{n, i}\right|+\frac{m}{b}\left|r_{n+1}-r_{n}\right| M_{2} .
$$

Hence we have from (3.5)-(3.7) and (3.18) that

$$
\begin{aligned}
& \left\|z_{n+1}-z_{n}\right\|-\left\|x_{n+1}-x_{n}\right\| \\
& \leq \frac{(\rho-1) \alpha_{n+1}}{1-\beta_{n+1}}\left\|x_{n+1}-x_{n}\right\|+\frac{\gamma_{n+1}}{1-\beta_{n+1}}\left(2 M \sum_{i=1}^{N}\left|\alpha_{n+1, i}-\alpha_{n, i}\right|+\frac{m}{b}\left|r_{n+1}-r_{n}\right| M_{2}\right) \\
& \quad+\left|\frac{\alpha_{n+1}}{1-\beta_{n+1}}-\frac{\alpha_{n}}{1-\beta_{n}}\right|\left\{\left\|f\left(x_{n}\right)\right\|+\left\|y_{n}\right\|\right\}+\frac{\gamma_{n+1}}{1-\beta_{n+1}}\left|\lambda_{n+1}-\lambda_{n}\right|\left\|x_{n}-W_{n} x_{n}\right\| .
\end{aligned}
$$


Notice conditions (ii) and (iii), (iv), we have that

$$
\limsup _{n \rightarrow \infty}\left(\left\|z_{n+1}-z_{n}\right\|-\left\|x_{n+1}-x_{n}\right\|\right)=0
$$

Hence we have from Lemma 2.4 that

$$
\limsup _{n \rightarrow \infty}\left\|z_{n}-x_{n}\right\|=0
$$

Therefore we have that

$$
\left\|x_{n+1}-x_{n}\right\|=\left|1-\beta_{n}\right|\left\|z_{n}-x_{n}\right\| \rightarrow 0 .
$$

Finally we show that $\left\{x_{n}\right\}$ converges strongly to $p \in F \cap C_{\min }$. Because $\mu_{n}\left\|x_{n}-p\right\|^{2}=$ inf $_{y \in C} \mu_{n}\left\|x_{n}-y\right\|^{2}$, we have from Lemma 2.1 that

$$
\mu_{n}\left\langle f(p)-p, J\left(x_{n}-p\right)\right\rangle \leq 0
$$

Due to the norm-weak* uniform continuity of the duality mapping $J$, it follows from (3.20) that

$$
\lim _{n \rightarrow \infty}\left(\left\langle f(p)-p, J\left(x_{n+1}-p\right)\right\rangle-\left\langle f(p)-p, J\left(x_{n}-p\right)\right\rangle\right)=0 .
$$

Hence, the sequence $\left\{\left\langle f(p)-p, J\left(x_{n}-p\right)\right\rangle\right\}$ satisfies the conditions of Lemma 2.2. As a result, we must have

$$
\limsup _{n \rightarrow \infty}\left\langle f(p)-p, J\left(x_{n+1}-p\right)\right\rangle \leq 0
$$

On the other hand, since $f$ is contractive with a contraction coefficient $\rho \in(0,1)$, we have from (3.3), (3.4) and Lemma 2.5 that

$$
\begin{aligned}
& \left\|x_{n+1}-p\right\|^{2} \\
& \quad=\left\|\alpha_{n}\left(f\left(x_{n}\right)-p\right)+\beta_{n}\left(x_{n}-p\right)+\gamma_{n}\left(y_{n}-p\right)\right\|^{2} \\
& \quad \leq\left\|\beta_{n}\left(x_{n}-p\right)+\gamma_{n}\left(y_{n}-p\right)\right\|^{2}+2 \alpha_{n}\left\langle f\left(x_{n}\right)-p, J\left(x_{n+1}-p\right)\right\rangle \\
& \quad \leq\left(1-\alpha_{n}\right)^{2}\left\|x_{n}-p\right\|^{2}+2 \alpha_{n}\left\{f\left(x_{n}\right)-f(p), J\left(x_{n+1}-p\right)\right\rangle+2 \alpha_{n}\left\langle f(p)-p, J\left(x_{n+1}-p\right)\right\rangle \\
& \quad \leq\left(1-\alpha_{n}\right)^{2}\left\|x_{n}-p\right\|^{2}+2 \rho \alpha_{n}\left\|x_{n}-p\right\|\left\|x_{n+1}-p\right\|+2 \alpha_{n}\left\langle f(p)-p, J\left(x_{n+1}-p\right)\right\rangle \\
& \quad \leq\left(1-\alpha_{n}\right)^{2}\left\|x_{n}-p\right\|^{2}+\rho \alpha_{n}\left[\left\|x_{n}-p\right\|^{2}+\left\|x_{n+1}-p\right\|^{2}\right]+2 \alpha_{n}\left\langle f(p)-p, J\left(x_{n+1}-p\right)\right\rangle,
\end{aligned}
$$

that is,

$$
\begin{aligned}
\left\|x_{n+1}-p\right\|^{2} \leq & {\left[1-\frac{2(1-\rho) \alpha_{n}}{1-\rho \alpha_{n}}\right]\left\|x_{n}-p\right\|^{2}+\frac{2 \alpha_{n}}{1-\rho \alpha_{n}}\left\langle f(p)-p, J\left(x_{n+1}-p\right)\right\rangle } \\
& +\frac{\alpha_{n}^{2}}{1-\rho \alpha_{n}}\left\|x_{n}-p\right\|^{2} .
\end{aligned}
$$


Let $\theta_{n}=\frac{2(1-\rho) \alpha_{n}}{1-\rho \alpha_{n}}, \sigma_{n}=\frac{2 \alpha_{n}}{1-\rho \alpha_{n}}\left\langle f(p)-p, J\left(x_{n+1}-p\right)\right\rangle+\frac{\alpha_{n}^{2}}{1-\rho \alpha_{n}}\left\|x_{n}-p\right\|^{2}$. Since $\left\{x_{n}\right\}$ is bounded, according to Lemma 2.3 and formula (3.23), we have that $\lim _{n \rightarrow \infty}\left\|x_{n}-p\right\|=0$, i.e., the sequence $\left\{x_{n}\right\}$ converges strongly to a common fixed point of $T_{i}: C \rightarrow C, i=1,2, \ldots, m$.

Theorem 3.2 Let $C$ be a nonempty closed convex subset of a uniformly smooth strictly convex real Banach space E. Let $\left\{T_{i}: C \rightarrow C, i=1,2, \ldots, m\right\}$ be a finite family of continuous pseudo-contractive mappings, for each bounded sequence $x_{n}$ and for each Banach limit $\mu_{n}$, $C_{\min }$ is defined as (2.2) satisfying $F \cap C_{\min } \neq \varnothing, f: C \rightarrow C$ is a contraction with a contraction coefficient $\rho \in(0,1)$. The mappings $T_{i r_{n}}$ and $W_{n}$ are defined as (3.1) and (3.2), respectively. Let $\left\{x_{n}\right\}$ be a sequence generated by $x_{0} \in C$

$$
\left\{\begin{array}{l}
y_{n}=\lambda_{n} x_{n}+\left(1-\lambda_{n}\right) W_{n} x_{n} \\
x_{n+1}=\alpha_{n} f\left(x_{n}\right)+\beta_{n} x_{n}+\gamma_{n} y_{n},
\end{array}\right.
$$

where $\lambda_{n} \in[0,1],\left\{\alpha_{n}\right\},\left\{\beta_{n}\right\},\left\{\gamma_{n}\right\}$ are sequences of nonnegative real numbers in $[0,1]$ and

(i) $\alpha_{n}+\beta_{n}+\gamma_{n}=1, n \geq 0$;

(ii) $\lim _{n \rightarrow \infty} \alpha_{n}=0, \sum_{n=1}^{\infty} \alpha_{n}=\infty$;

(iii) $\lim _{n \rightarrow \infty} \lambda_{n}=1$;

(iv) $\lim _{n \rightarrow \infty}\left(\alpha_{n, i}-\alpha_{n-1, i}\right)=0 ; \lim _{n \rightarrow \infty}\left|r_{n+1}-r_{n}\right|=0$.

Then the sequence $\left\{x_{n}\right\}$ converges strongly to a common fixed point of $\left\{T_{i}: C \rightarrow C, i=\right.$ $1,2, \ldots, m\}$.

Proof Take $p \in F \cap C_{\min }$, from (3.24) we can obtain

$$
\begin{aligned}
\left\|x_{n+1}-x_{n}\right\| & =\left\|\alpha_{n} f\left(x_{n}\right)+\beta_{n} x_{n}+\gamma_{n} y_{n}-p\right\| \\
& \leq \alpha_{n}\left\|f\left(x_{n}\right)-x_{n}\right\|+\gamma_{n}\left(1-\lambda_{n}\right)\left\|W_{n} x_{n}-x_{n}\right\| .
\end{aligned}
$$

Notice the boundedness of the sequences $\left\{x_{n}\right\}$ and $\left\{W_{n} x_{n}\right\}$. According to conditions (ii) and (iii), we have $\lim _{n \rightarrow \infty}\left\|x_{n+1}-x_{n}\right\|=0$. Similar to Theorem 3.1, we can obtain the result.

If in Theorem 3.1 and Theorem 3.2 we let $f: \equiv u \in C$ be a constant mapping, we have the following corollary.

Corollary 3.3 Let $C$ be a nonempty closed convex subset of a uniformly smooth strictly convex real Banach space E. Let $\left\{T_{i}: C \rightarrow C, i=1,2, \ldots, m\right\}$ be a finite family of continuous pseudo-contractive mappings, for each bounded sequence $x_{n}$ and for each Banach limit $\mu_{n}$, $C_{\min }$ is defined as (2.2) satisfying $F \cap C_{\min } \neq \varnothing$. The mappings $T_{i r_{n}}$ and $W_{n}$ are defined as (3.1) and (3.2), respectively. Let $\left\{x_{n}\right\}$ be a sequence generated by $x_{0} \in C$

$$
\left\{\begin{array}{l}
y_{n}=\lambda_{n} x_{n}+\left(1-\lambda_{n}\right) W_{n} x_{n} \\
x_{n+1}=\alpha_{n} u+\beta_{n} x_{n}+\gamma_{n} y_{n}
\end{array}\right.
$$

where $\lambda_{n} \in[0,1],\left\{\alpha_{n}\right\},\left\{\beta_{n}\right\},\left\{\gamma_{n}\right\}$ are the sequences of nonnegative real numbers in $[0,1]$ and 
(i) $\alpha_{n}+\beta_{n}+\gamma_{n}=1, n \geq 0$;

(ii) $\lim _{n \rightarrow \infty} \alpha_{n}=0, \sum_{n=1}^{\infty} \alpha_{n}=\infty$;

(iii) $0<\liminf _{n \rightarrow \infty} \lambda_{n} \leq \lim \sup _{n \rightarrow \infty} \lambda_{n}<1$ or $\lim _{n \rightarrow \infty} \lambda_{n}=1$;

(iv) $\lim _{n \rightarrow \infty}\left(\alpha_{n, i}-\alpha_{n-1, i}\right)=0 ; \lim _{n \rightarrow \infty}\left|r_{n+1}-r_{n}\right|=0$.

Then the sequence $\left\{x_{n}\right\}$ converges strongly to a common fixed point of $\left\{T_{i}: C \rightarrow C, i=\right.$ $1,2, \ldots, m\}$.

Theorem 3.4 Let $C$ be a nonempty closed convex subset of a uniformly smooth strictly convex real Banach space E. Let $T: C \rightarrow C$ be a continuous pseudo-contractive mapping for each bounded sequence $x_{n}$ and for each Banach limit $\mu_{n}, C_{\min }$ be defined as (2.2) satisfying $F(T) \cap C_{\min } \neq \varnothing, f: C \rightarrow C$ be a contraction with a contraction coefficient $\rho \in(0,1)$. Mapping $T_{r_{n}}$ is defined as follows: $x \in C, r_{n} \in(0, \infty)$

$$
T_{r_{n}}(x)=\left\{z \in C:\langle j(y-z), T z\rangle-\frac{1}{r_{n}}\left\langle j(y-z),\left(1+r_{n}\right) z-x\right\rangle \leq 0, \forall y \in C\right\} .
$$

Let $\left\{x_{n}\right\}$ be a sequence generated by $x_{0} \in C$

$$
\left\{\begin{array}{l}
y_{n}=\lambda_{n} x_{n}+\left(1-\lambda_{n}\right) T_{r_{n}} x_{n}, \\
x_{n+1}=\alpha_{n} f\left(x_{n}\right)+\beta_{n} x_{n}+\gamma_{n} y_{n},
\end{array}\right.
$$

where $\lambda_{n} \in[0,1]$ and $\left\{\alpha_{n}\right\},\left\{\beta_{n}\right\},\left\{\gamma_{n}\right\}$ are the sequences of nonnegative real numbers in $[0,1]$ and

(i) $\alpha_{n}+\beta_{n}+\gamma_{n}=1, n \geq 0$;

(ii) $\lim _{n \rightarrow \infty} \alpha_{n}=0, \sum_{n=1}^{\infty} \alpha_{n}=\infty$;

(iii) $0<\liminf _{n \rightarrow \infty} \lambda_{n} \leq \lim \sup _{n \rightarrow \infty} \lambda_{n}<1$ or $\lim _{n \rightarrow \infty} \lambda_{n}=1$;

(iv) $\lim _{n \rightarrow \infty}\left|r_{n+1}-r_{n}\right|=0$.

Then the sequence $x_{n}$ converges strongly to a fixed point of $T$.

Proof Putting $\alpha_{n, i}=1$ in (3.2), we have $W_{n}=T_{r, n}$; from Lemma 2.4 and Theorems 3.1 and 3.2, we can obtain the result.

\section{Competing interests}

The author declares that they have no competing interests.

\section{Acknowledgements}

Article is supported by the National Science Foundation of China (11001287) and Natural Science Foundation Project of Chongqing (CSTC, 2012jjA00039) and Science and Technology Research Project of Chongqing Municipal Education Commission (KJ130712, KJ130731).

Received: 25 June 2013 Accepted: 13 March 2014 Published: 28 Mar 2014

\section{References}

1. Chidume, CE, Mutangadura, SA: An example of the Mann iteration method for Lipschitz pseudocontractions. Proc. Am. Math. Soc. 129, 2359-2363 (2001)

2. Ishikawa, S: Fixed points by a new iteration method. Proc. Am. Math. Soc. 44, 147-150 (1974)

3. Zhou, HY, Wei, L, Cho, YJ: Strong convergence theorems on an iterative method for a family of finite nonexpansive mappings in reflexive Banach spaces. Appl. Math. Comput. 173, 196-212 (2006)

4. Halpern, B: Fixed points of nonexpanding maps. Bull. Am. Math. Soc. 73, 957-961 (1967)

5. Yao, Y: A general iterative method for a finite family of nonexpansive mappings. Nonlinear Anal. 66, 2676-2687 (2007)

6. Yao, Y, Chen, R, Yao, JC: Strong convergence and certain conditions for modified Mann iteration. Nonlinear Anal. 68, 1687-1693 (2008) 
7. Chang, SS, Joseph Lee, HW, Chan, CK: On Reich's strong convergence theorem for asymptotically nonexpansive mappings in Banach spaces. Nonlinear Anal. 66, 2364-2374 (2007)

8. Xu, HK: Viscosity approximation methods for nonexpansive mappings. J. Math. Anal. Appl. 298, $279-291$ (2004)

9. Song, Y: A new sufficient condition for the strong convergence of Halpern type iterations. Appl. Math. Comput. 198, 721-728 (2008)

10. Suzuki, T: Strong convergence of Krasnoselskii and Mann's type sequences for one-parameter nonexpansive semigroups without Bochner integrals. J. Math. Anal. Appl. 305, 227-239 (2005)

11. Suzuki, T: Strong convergence of Krasnoselskii and Mann's type sequences for one-parameter non-expansive semigroups without Bochner integrals. J. Math. Anal. Appl. 305, 227-239 (2005)

12. Atsushiba, S, Takahashi, W: Strong convergence theorems for a finite family of non-expansive mappings and applications. Indian J. Math. 41, 435-453 (1999)

13. Xu, HK: An iterative approach to quadratic optimization. J. Optim. Theory Appl. 116, 659-678 (2003)

14. Lou, J, Zhang, L, He, Z: Viscosity approximation methods for asymptotically nonexpansive mappings. Appl. Math. Comput. 203, 171-177 (2008)

15. Ceng, LC, Xu, HK, Yao, JC: The viscosity approximation method for asymptotically nonexpansive mappings in Banach spaces. Nonlinear Anal. 69, 1402-1412 (2008)

16. Song, $Y$, Chen, R: Strong convergence theorems on an iterative method for a family of finite non-expansive mappings. Appl. Math. Comput. 180, 275-287 (2006)

17. Zegeye, $\mathrm{H}$, Shahzad, $\mathrm{N}$ : Strong convergence for monotone mappings and relatively weak nonexpansive mappings. Nonlinear Anal. 70, 2707-2716 (2009)

18. Wen, DJ, Deng, L: Approximate methods and theorems of common fixed-points for a finite family of nonexpansive mappings. Acta Math. Sci. 32(3), 540-546 (2012)

19. Moudifi, A: Viscosity approximation methods for fixed point problems. J. Math. Anal. Appl. 241, 46-55 (2000)

20. Zegeye, $\mathrm{H}$, Shahzad, N: Strong convergence of an iterative method for pseudo-contractive and monotone mappings. J. Glob. Optim. 54, 173-184 (2012)

21. Takahashi, W: Non-linear Functional Analysis-Fixed Point Theory and Its Applications. Yokohama Publishers, Yokohama (2000)

22. Takahashi, W, Toyoda, M: Weak convergence theorems for nonexpansive mappings and monotone mappings. J. Optim. Theory Appl. 118, 417-428 (2003)

23. Plubtieng, S, Punpaeng, R: Fixed-point solutions of variational inequalities for nonexpansive semigroups in Hilbert spaces. Math. Comput. Model. 48, 279-286 (2008)

24. Zegeye, $\mathrm{H}$ : An iterative approximation method for a common fixed point of two pseudocontractive mappings. ISRN Math. Anal. (2011). doi:10.5402/2011/621901

10.1186/1029-242X-2014-124

Cite this article as: Tang: Strong convergence theorems on a viscosity approximation method for a finite family of pseudo-contractive mappings in Banach spaces. Journal of Inequalities and Applications 2014, 2014:124

\section{Submit your manuscript to a SpringerOpen ${ }^{\circ}$ journal and benefit from:}

- Convenient online submission

- Rigorous peer review

- Immediate publication on acceptance

Open access: articles freely available online

- High visibility within the field

- Retaining the copyright to your article 